\title{
UM MÉTODO QUANTITATIVO PARA AVALIAÇÃO DA SATISFAÇÃO DOS CLIENTES ${ }^{1}$
}

\author{
Fátima de Souza Freire
}

Professora Adjunto III da Universidade Federal do Ceará - UFC

Chefe do Departamento de Contabilidade da Universidade Federal do Ceará - UFC

Doutora em Economia Industrial pela Université des Sciences Sociales, Toulouse, França

E-mail: ffreire@ufc.br

\section{Vicente Lima Crisóstomo}

Prof. Assistente II do Departamento de Contabilidade da Universidade Federal do Ceará - UFC

Mestre em Ciência da Computação pela Universidade Federal de Santa Catarina - UFSC

E-mail: vlc@ufc.br

\section{Ducineli Régis Botelho}

Mestranda em Contabilidade da Universidade de Brasília - UnB

Bacharel em Ciências Contábeis pela Universidade Federal do Ceará - UFC

E-mail:duci@botelho.eti.br

RESUMO

Há premência em se sistematizar esquemas para extrair informações dos novos indicadores que estão sendo empregados nos modernos métodos de avaliação do desempenho operacional das empresas. $\mathrm{Na}$ ampla maioria destes, a perspectiva do cliente é sempre levada em consideração. Entretanto, as pesquisas junto a eles sempre fornecem informações qualitativas, embora constituam uma das áreas mais atuantes das empresas de pesquisa de mercado. Enfrentando o desafio de generalizar um modelo contábil para incorporação da avaliação quantitativa de ativos intangíveis de empresas que seja útil para a moderna caracterização dos seus desempenhos operacionais, propõe-se neste trabalho um esquema inovador para avaliar a satisfação dos clientes capaz de fornecer resultados quantitativos inequívocos. Os indicadores que o método proposto utiliza provêm de enquêtes bem definidas, realizadas junto aos clientes, e possibilitam o advento de índices que caracterizarão ativos e passivos das empresas relativos à satisfação dos clientes.

Palavras-chave: Estratégias de gestão, Indicadores de satisfação, Avaliação dos clientes, Método quantitativo.
ABSTRACT

It is a matter of certain urgency to formalize rules with a view to extracting information from the new indicators that are used in modern enterprise evaluation methods. In most situations, the client's point of view is always taken into consideration. However, in spite of being one of the most active areas for market research companies, client inquiries always provide qualitative information. Facing the challenge of generalizing an accounting model that incorporates the quantitative evaluation of companies' intangible assets so as to be useful for improving their performance, this paper proposes an innovative method for evaluating client satisfaction. The elements used in this new method come from inquiries that are regularly held among clients. The obtained results provide the calculation of indices that characterize companies' assets and liabilities and are related to client satisfaction.

Keywords: Administration strategies, Satisfaction Indicators, Clients' Evaluation, Quantitative Method. 


\section{INTRODUÇÃO}

No mundo dos negócios, o lema "o cliente tem sempre razão" é de aceitação máxima, generalizada. Compreende-se que o sucesso de uma atividade empresarial seja indissociavelmente ligado à busca da satisfação do cliente e, por isto, esta guia, de uma forma ou de outra e desde tempos imemoriais, muito das estratégias empresariais. Se fosse trivial detectar o que tem de ser feito para a satisfação do cliente em termos de produto como, por exemplo, atendimento, os caminhos para uma empresa atingir o sucesso seriam bem menos tortuosos. É por isto que atividades relacionadas com a opinião do cliente sempre se encontram presentes em todos os métodos de avaliação do desempenho operacional das empresas.

Praticamente até os anos oitenta, as atividades empresariais eram desenvolvidas num ambiente moldado por relatórios financeiros, sob a égide predeterminante do modelo contábil tradicional. Não que deixasse de se atentar para ativos intangíveis (daí a possibilidade da existência do goodwill nos relatórios), mas no império da contabilidade financeira tradicional é extremamente frustrante tentar considerar-se o papel de ativos intangíveis, recursos humanos, clientela e marcas, durante a avaliação do desempenho operacional das empresas, e para o estabelecimento do quadro absolutamente necessário de estratégia empresarial futura.

Com a cada vez mais clara dicotomia entre a contabilidade financeira desenvolvida desde há séculos para transações isoladas entre empresas independentes, e a crescente importância da contribuição dos ativos intangíveis para o sucesso empresarial, deu-se na década de noventa o advento de vários métodos modernos para avaliação do desempenho empresarial: balanced scorecard (Kaplan e Norton, 1992); Effective Progress and Performance Measurement (Adams e Roberts, 1993); Quantum (Hronec e A. Andersen Co., 1993) etc. De uma forma ou de outra, eles fazem uso de indicadores relativos a ativos intangíveis lado a lado com os indicadores contábeis e financeiros tradicionais. A título de exemplo, pode-se abordar o caso do balanced scorecard, que tem como objetivo permitir uma gestão eficaz da performance organizacional, baseando-se na visão e estratégia da empresa e traduzindo-a em indicadores de performance financeiros e não financeiros (Kaplan e Norton, 1997).

No balanced scorecard, embora os indicadores internos a partir de dados quantitativos (custos, balanços, produção, rendimento etc.) quanto à performance da empresa possibilitem que o gestor decida na geração dos bens e serviços, a opinião dos agentes econômicos deve ser também levada em consideração, pois a necessidade dos clientes e fornecedores, a satisfação dos empregados, bem como ações do governo poderão levar a empresa para outro caminho.

Nota-se que em todos os métodos modernos para avaliação do desempenho empresarial, a análise das empresas sob a perspectiva do cliente é elemento relevante, mas é realizada de forma qualitativa, em geral através da elaboração e aplicação de pesquisas de opinião junto aos clientes. No entanto, são em geral falhos em converter/sistematizar as utilíssimas respostas obtidas pelas enquêtes em relatórios da satisfação do cliente que poderiam ser reconhecidos como uma extensão/generalização daqueles da contabilidade financeira, os quais deveriam conter índices para caracterizar ativos e passivos das empresas relativos à satisfação dos clientes.

Baseado em informações extraídas dos agentes econômicos, surge então mais um novo método de mensuração da performance das empresas: o Balanço da Perspectiva Abrangente dos Clientes (BPAC). Este método possibilita que a empresa verifique a sua performance social-econômica por meio da análise de indicadores baseados em enquêtes realizadas junto aos mesmos.

O presente trabalho tem como objetivo principal apresentar um método quantitativo para avaliação da satisfação dos clientes. Ele possibilita a análise da satisfação dos clientes através de índices que caracterizarão ativos e passivos de satisfação.

$\mathrm{Na}$ seção 2, encontra-se uma abordagem sobre o balanced scorecard e sua ligação com o método proposto. Na seção 3, a finalidade, os indicadores e a preparação da planilha contábil generalizada de mensuração da satisfação do cliente são apresentados. Uma breve discussão é realizada na seção 4 sobre a forma de ampliar o esquema desenvolvido para incorporar todos os parâmetros importantes 
para a empresa, no que tange à perspectiva do cliente em uma planilha síntese de cunho contábil. Este trabalho se encerra na seção 5, onde são apresentadas as conclusões e perspectivas de desenvolvimento futuro do método quantitativo de avaliação da satisfação do cliente nele apresentado.

\section{O BALANCED SCORECARD}

Inicialmente desenvolvido pelo Dr. Robert Kaplan e David Norton (1997), o balanced scorecard é uma filosofia prática e inovadora de gestão da performance das empresas e organi- zações. O objetivo da sua implementação é permitir uma gestão eficaz da performance organizacional, baseando-se na visão e estratégia da empresa e traduzindo-a em indicadores financeiros e não financeiros.

Contrastando com os sistemas de medição tradicionais baseados exclusivamente na vertente financeira, o balanced scorecard direciona a organização para o seu sucesso no futuro, definindo quais os objetivos a atingir e medindo a sua performance a partir de quatro perspectivas distintas (ver Quadro 1): financeira, de clientes ou mercado, de processos internos e de aprendizado e crescimento.

\section{QUADRO 1 - MEDIDAS GENÉRICAS DO BALANCED SCORECARD}

\begin{tabular}{|l|l|}
\hline $\begin{array}{l}\text { Perspectiva } \\
\text { Financeira }\end{array}$ & Medidas Genéricas \\
\hline Do cliente & Satisfação, retenção, participação de mercado e participação de conta. \\
\hline Interna & Qualidade, tempo de resposta, custo e lançamentos de novos produtos. \\
\hline $\begin{array}{l}\text { Aprendizado e } \\
\text { crescimento }\end{array}$ & Satisfação dos funcionários e disponibilidade dos sistemas de informação. \\
\hline
\end{tabular}

Fonte: Robert S. Kaplan e David P. Norton. 1997.

Segundo Kaplan e Norton (1997), no caso específico da perspectiva de clientes, busca-se encontrar medidas genéricas como: a participação no mercado, retenção de clientes, aquisição de novos clientes, rentabilidade dos clientes, qualidade do produto, relacionamento com clientes, imagem e reputação, e satisfação dos clientes.

"Nos últimos anos a ênfase nos clientes e na qualidade dos processos levou muitas empresas a acompanhar e comunicar medidas de satisfação e reclamações dos clientes, níveis de defeito dos produtos e processos, e descumprimento dos prazos de entrega. Na França as empresas desenvolveram e vêm utilizando, há mais de duas décadas, o tableau de bord, um painel de indicadores - chaves do sucesso organizacional. $O$ tableau de bord tem por finalidade ajudar os funcionários a pilotar a empresa mediante a identificação dos fatores chaves de sucesso, principalmente os que podem ser medidos por variáveis físicas" (Norton e Kaplan, 1996). Logo, o método de avaliação, proposto neste trabalho, possibilita a mensuração da medida genérica "satisfação dos clientes".

\section{FINALIDADE, INDICADORES E PLANILHA PARA AVALIAÇÃO DA SATISFAÇÃO DOS CLIENTES}

A prospecção da satisfação do cliente é medida sine qua non na investigação do desempenho empresarial. A partir das informações dela advinda, medidas em relação ao cliente podem ser tomadas visando a sua retenção, a aquisição de novos, a determinação da lucratividade relacionada, e sua participação nos segmentos-alvo (Kaplan e Norton, 1997). Pesquisas de opinião realizadas através da aplicação de enquêtes são os meios empregados para obtenção do feedback do cliente em relação aos produtos, atividades, atendimento, pontualidade, etc. das empresas. Elas constituem uma complicada, mas ativa área da prospecção do mercado, produzindo relatórios bastante pertinentes - mas tendo por base indicadores qualitativos - que podem caracterizar de certa forma o nível de satisfação dos clientes.

A finalidade do método para avaliação da satisfação dos clientes aqui proposto é possibilitar que a empresa extraia informações quantitativas das pesquisas de opinião normalmente aplicadas junto aos 
clientes. A ênfase é a determinação de índices conseqüentes dos itens das enquêtes que possam ser caracterizados univocamente como ativo ou passivo da empresa no que concerne à satisfação dos clientes. Claro que as respostas dos clientes aos itens das enquêtes são, de uma forma ou de outra, mister das atividades desenvolvidas nas empresas que estão associadas àqueles itens. Conseqüentemente, na construção de ativos ou passivos das empresas relativos à satisfação dos clientes, deve-se considerar tanto as componentes conseqüentes da satisfação dos clientes (advindas dos itens das enquêtes aplicadas) como as componentes geratrizes (advindas dos meios utilizados pelas empresas para satisfação dos clientes).

Por mais intensas e bem programadas que tenham sido as atividades de uma empresa para satisfazer o cliente em um determinado aspecto, não necessariamente elas terão sucesso. Assim, uma componente geratriz da satisfação do cliente pode contribuir de forma positiva para o que seria uma espé- cie de contribuição ao ativo da empresa relativo à satisfação dos clientes. Por outro lado, a percepção da mesma, avaliada através da componente conseqüente, poderia indicar que os meios utilizados pela empresa para satisfação dos clientes não produziram o retorno desejado, de forma que se poderia à implementação destes meios associar uma espécie de contribuição ao passivo da empresa relativa à satisfação dos clientes.

Cada indicador quantitativo relativo à satisfação do cliente seria "calculado" em acordo com os resultados das enquêtes tradicionais aplicadas junto a eles próprios (componente conseqüente), e com uma avaliação interna pelas empresas, dos meios que utilizaram para obtenção de sucesso no que concerne à satisfação do cliente (componente geratriz). Por exemplo, a enquête determinaria uma componente conseqüente da satisfação dos clientes em relação a um determinado item, que seria obtida através das respostas da clientela à seguinte pergunta:

Como avalia o atendimento ao cliente dispensado pela empresa durante 0 ano?

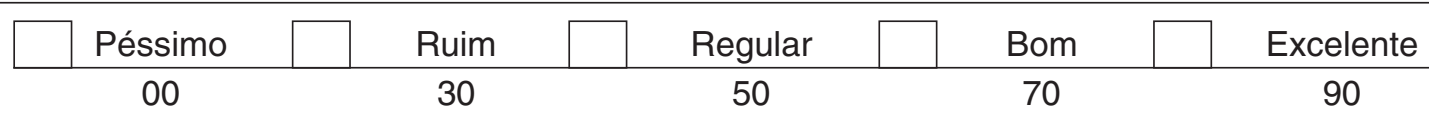

A componente conseqüente do indicador Atendimento ao Cliente $\left(A C_{c c}\right)$ seria calculada através da seguinte expressão:

$$
A C_{c c}=\frac{\sum_{\mathrm{i}=1}^{n} N R_{i} V_{i}}{N T R}
$$

onde $N R_{i}$ seria o número de respostas do $i$-ésimo item, $V_{i}$ o valor $(00 \ldots 90)$ associado a cada item, e NTR o número total de respostas da pergunta feita à clientela da empresa relativa ao indicador Atendimento ao Cliente.

A componente geratriz do indicador Atendimento da junto aos funcionários da empresa que ao cliente $\left(A C_{c g}\right)$ seria determinada, por exemplo, por atendem aos clientes, que teriam perguntas meio dos resultados de uma enquête interna realiza- do tipo:

\section{Como avalia o atendimento que dispensou ao cliente da empresa durante 0 ano?}

\begin{tabular}{|c|cccccc|c|}
\hline Péssimo & & Ruim & & Regular & & Bom & Excelente \\
\hline 00 & 30 & 50 & 70 & 90
\end{tabular}


A componente geratriz do indicador Atendimento ao Cliente seria calculada através de expressão semelhante àquela utilizada para o cálculo da componente conseqüente.

Resulta então que o indicador final relativo ao Atendimento ao Cliente de um determinado período (anual ou mensal, por exemplo) seria a média $\left(A C_{c g}+A C_{c c}\right) / 2$. A performance positiva (Ativo) ou negativa (Passivo) da empresa em relação ao Atendimento ao Cliente [ou seja, avanço ou retrocesso no Atendimento ao Cliente por parte da empresa], em relação a este indicador, seria obtida simplesmente pela variação temporal (anual ou mensal) do índice de satisfação ao cliente relativa ao indicador. No caso do Atendimento ao Cliente, tem-se

$\Delta I S C_{A C}=\Delta I S C_{A C}($ período $x+1)-\Delta I S C_{A C}($ período $x)$, onde ISC = Índice de Satisfação de Cliente

Claro que todos os questionamentos, realizados tanto na enquête interna como naquela junto aos clientes, estariam diretamente relacionados com algum tipo de informação necessária para avaliação do atendimento ao cliente, por parte da empresa. Devido à abrangência e diversidade dos itens das duas enquêtes, seria então necessária a elaboração de uma "fórmula de cálculo" específica para cada indicador prospectivo da satisfação do cliente arrolado na planilha do método quantitativo de avaliação da satisfação dos clientes. ${ }^{2}$

Como todas as questões das enquêtes para os clientes e para os agentes internos da empresa não têm a mesma importância para averiguação da satisfação do cliente por parte da empresa, elas estabeleceriam de modo natural uma ordem de prioridade à qual seria associado um fator de ponderação, cujo valor (ou peso) refletiria sua importância quanto à satisfação do cliente por parte da empresa, mas de forma que a soma dos mesmos fosse 100. As enquêtes seriam aplicadas anualmente ou mensalmente, por exemplo, e proporcionariam uma visão da satisfação do cliente por parte da empresa naquele período, e também se as ações desenvolvidas no seio interno para satisfação dos clientes encontraram eco por parte dos mesmos. A comparação dos resultados de dois períodos subsequentes permitiria detectar se houve avanço ou retrocesso na satisfação do cliente por parte da empresa, no que tange a cada questão da enquête. Uma configuração de ativo ou passivo de satisfação do cliente seria então associada a cada questão da enquête ao multiplicar a porcentagem de avanço ou retrocesso pelo valor (ou peso) relativo da questão.

A soma de todos os ativos de satisfação dos clientes de cada enquête determinaria uma espécie de grau de satisfação dos clientes GSC (avaliação positiva da satisfação dos clientes) advindo das enquêtes prospectivas realizadas, da mesma forma que a soma de todos os passivos de satisfação dos clientes de cada enquête forneceria uma espécie de grau de insatisfação dos clientes GIC (avaliação negativa da satisfação dos clientes). Os graus de satisfação e insatisfação dos clientes seriam caracterizados por valores em unidades de satisfação dos clientes (USC), e a diferença entre os mesmos seria então um déficit ou superávit de satisfação do cliente detido pela empresa, em termos desta mesma unidade.

A Tabela 1 apresenta um exemplo dos indicadores de satisfação dos clientes resultantes de uma hipotética enquête realizada junto à clientela de uma empresa. Deve-se destacar que os indicadores nas colunas dos resultados refletem o esquema utilizado na análise da enquête por parte de quem está operacionalizando o método quantitativo de avaliação da satisfação dos clientes. ${ }^{3}$

\footnotetext{
$2 \mathrm{O}$ "cálculo" relativo ao indicador Atendimento ao Cliente apresentado neste trabalho deve ser reconhecido apenas como um exemplo das diferentes possibilidades que se abrem com o Método Quantitativo de Avaliação da Satisfação dos Clientes na forma aqui proposta.

3 Os indicadores utilizados na Tabela 1 são semelhantes aos utilizados na Fig. 4-6, p. 87, de A Estratégia em Ação (Kaplan e Norton, 1997).
} 
Tabela 1 - Resultados da enquête relativa aos indicadores de satisfação dos clientes, aplicada junto aos clientes de uma empresa hipotética.

\begin{tabular}{|c|c|c|c|c|c|c|c|}
\hline \multirow[t]{2}{*}{$\begin{array}{l}\text { Indicadores Satisfação de } \\
\text { Clientes }\end{array}$} & \multirow[t]{2}{*}{ PR } & \multirow[t]{2}{*}{ FP ou PE } & \multicolumn{2}{|c|}{ RESULTA (\%) } & \multirow[t]{2}{*}{$E(\%)$} & \multirow[t]{2}{*}{ AAC } & \multirow[t]{2}{*}{ PAC } \\
\hline & & & 1998 & 1999 & & & \\
\hline Segurança & 1 & $5 / 100$ & 97,98 & 98,70 & 0,72 & 3,60 & \\
\hline Cumprimento dos Cronogramas & 2 & $10 / 100$ & 80,73 & 80,03 & $-0,70$ & & 7,00 \\
\hline Relação Horas Trabalhadas/Defeitos & 3 & $10 / 100$ & 74,17 & 71,21 & $-2,96$ & & 29,60 \\
\hline Apres. Procedimentos Tempo Hábil & 4 & $5 / 100$ & 66,04 & 62,53 & $-3,51$ & & 17,55 \\
\hline Rev. Mínima Proc. Apresentados & 5 & $5 / 100$ & 73,65 & 72,39 & $-1,26$ & & 6,30 \\
\hline Atendimento ao Cliente & 6 & $10 / 100$ & 82,52 & 81,69 & $-0,83$ & & 8,30 \\
\hline Honest. Receptividade Fornecedores & 7 & $5 / 100$ & 82,51 & 81,53 & $-0,98$ & & 4,90 \\
\hline Flexibilidade & 8 & $5 / 100$ & 75,10 & 73,25 & $-1,85$ & & 9,25 \\
\hline Cumprimento dos Contratos & 9 & $5 / 100$ & 79,07 & 78,33 & $-0,74$ & & 3,70 \\
\hline Serviços de Engenharia & 10 & $5 / 100$ & 73,23 & 72,09 & $-1,14$ & & 5,70 \\
\hline Relação Custo-Benefício & 11 & $10 / 100$ & 64,83 & 62,53 & $-2,30$ & & 23,00 \\
\hline Padrão Equipamentos Oferecidos & 12 & $5 / 100$ & 69,33 & 67,17 & $-2,16$ & & 10,80 \\
\hline Qualidade do Pessoal & 13 & $5 / 100$ & 74,61 & 73,62 & $-0,99$ & & 4,95 \\
\hline Inovação Reduzir Custos & 14 & $5 / 100$ & 68,76 & 67,73 & $-1,03$ & & 5,15 \\
\hline Qualidade da Produção & 15 & $5 / 100$ & 80,37 & 80,75 & 0,38 & 1,90 & \\
\hline Relacionamento/Espírito Equipe & 16 & $5 / 100$ & 71,96 & 69,29 & $-2,67$ & & 13,35 \\
\hline \multicolumn{6}{|l|}{ Grau de Satisfação dos Clientes (USC) } & 5,50 & \\
\hline \multicolumn{7}{|c|}{ Grau de Insatisfação dos Clientes (USC) } & 149,55 \\
\hline \multicolumn{7}{|c|}{ Déficit na Satisfação dos Clientes da Empresa (USC) } & $(144,05)$ \\
\hline \multicolumn{6}{|c|}{ Totalização } & 5,50 & 5,50 \\
\hline
\end{tabular}

PR - Prioridade; FP - Fator de Ponderação; PE - peso; E(\%) - Avanço ou retrocesso da avaliação (\%); AAC - Ativo relacionado à Avaliação do Cliente; PAC - Passivo relacionado à Avaliação do Cliente; USC - Unidade de Satisfação dos Clientes.

\section{NOTAS:}

(i) A parte da planilha de 1999 relativa aos clientes foi implementada através de enquête junto a 2830 clientes, que de forma voluntária e anônima a responderam. Este número representa $23,24 \%$ da clientela da empresa.

(ii) A avaliação dos indicadores de satisfação dos clientes considera tanto a percepção da componente conseqüente quanto a componente geratriz dos clientes.

Uma análise da Tabela 1 mostra que o grau de satisfação dos clientes da empresa no ano de 1999 foi inferior ao de 1998, o que está caracterizado pela existência de um déficit de 144,05 USC (Unidades de Satisfação do Cliente) na satisfação dos clientes da empresa no biênio. No entanto, a satisfação dos clientes da empresa decaiu em quartoze indicadores, mas melhorou nos quesitos de Segurança e Qualidade de Produção. Por outro lado, a satisfação do cliente decresceu principalmente em relação às Ho- ras Trabalhadas/Defeitos, Apresentação de Procedimentos em Tempo Hábil, Revisão Mínima dos Procedimentos Apresentados, Serviços de Engenharia, e no Relacionamento/Espírito de Equipe (mais de $70 \%$ do passivo da empresa relativo à satisfação dos clientes está associada a estes cinco indicadores).

Como os procedimentos de atendimento aos clientes dependem das atividades desenvolvidas por cada empresa, os indicadores de satisfação dos clientes devem ser determinados para cada caso, as- 
sim como as enquêtes que serão aplicadas e as fórmulas para o cálculo do grau de satisfação ou insatisfação dos clientes relativa a um biênio.

A planilha do método, enquêtes e formulário devem ser retidos para tornar possível a determinação de ativos e passivos medidos em termos da unidade de satisfação do cliente (USC). No biênio, deve ser destacado que o advento inequívoco do status de ativo ou passivo em relação a um determinado indicador de satisfação dos clientes permite uma determinação precisa de ações estratégicas visando à correção de rumos (no caso de passivos) ou a sua manutenção/melhoria (no caso de ativos).

É conveniente deixar explícito que o método quantitativo para avaliação da satisfação dos clientes aqui apresentado constitui uma ampliação da sistemática utilizada para a produção dos relatórios de contabilidade financeira, claro que com adaptações que incorporam de forma clara e precisa a avaliação dos parâmetros intangíveis relacionados a uma mensuração da satisfação dos clientes. A planilha obtida configura-se um documento de caráter contábil em que a essência de sua preparação se remete às partidas dobradas, marco fundamental na Ciência Contábil.

\section{O BALANÇO DA PERSPECTIVA ABRANGENTE DO CLIENTE}

A perspectiva do cliente para as empresas não é só a de sua satisfação, mas compreende também sua fidelidade, retenção, captação e lucratividade (Kaplan e Norton, 1997). Portanto, avaliar o desempenho operacional das empresas no que tange à perspectiva do cliente não envolve somente a determinação da satisfação dos clientes, mas também a determinação dos resultados essenciais relativos à fidelidade, retenção, captação e lucratividade a eles associada. Métodos quantitativos similares podem ser implementados para estes itens, utilizando procedimentos similares aos desenvolvidos na seção anterior para avaliação quantitativa da satisfação dos clientes.

Claro que os indicadores de cada enquête devem refletir a necessidade do que se deseja apreender em relação à fidelidade, retenção, captação e lucratividade associada aos clientes. Através dos formulários específicos, os indicadores permitirão a ob- tenção de ativos ou passivos a eles associados que serão mensurados, por exemplo, em termos de unidade de fidelidade dos clientes (UFC), unidade de retenção dos clientes (URC), unidade de captação dos clientes (UCC) e unidade de lucratividade dos clientes (ULC).

As enquêtes realizadas para a obtenção de informações relativas à fidelidade, retenção, captação e lucratividade associada aos clientes - todas com suas peculiaridades - gerariam planilhas similares à Tabela 1. Os indicadores das tabelas difeririam consideravelmente entre si, mas cada um deles refletiria uma visão da fidelidade, retenção, captação e lucratividade associada aos clientes. A ordem de prioridade dos indicadores sociais seria característica de cada tipo de perspectiva do cliente, mas a cada indicador seria também associado um fator de ponderação, cujo valor (ou peso) refletiria sua importância quanto àquela perspectiva do cliente, mas de forma que a soma dos mesmos também fosse 100 .

As enquêtes seriam aplicadas anualmente, e proporcionariam uma visão da fidelidade, retenção, captação e lucratividade associada aos clientes da empresa naquele ano. A comparação dos resultados de dois anos permitiria detectar se houve avanço ou retrocesso da empresa em cada questão da enquête relativa ao tipo de perspectiva do cliente abordada. Cada planilha, construída relativa à fidelidade, retenção, captação e lucratividade associada aos clientes de uma empresa, seria na verdade uma generalização/adaptação de um balanço contábil para avaliação daquele intangível específico.

O Balanço da Perspectiva Abrangente dos Clientes (BPAC) de uma empresa seria, então, preparado através da utilização dos dados sobre a satisfação, fidelidade, retenção, captação e lucratividade dos clientes, determinados de acordo com os resultados das enquêtes que necessariamente seriam aplicadas. Claro que nem todos os itens da perspectiva dos clientes deveriam ter a mesma relevância!

Por isto, também tem que ser estabelecido um conjunto de prioridades no que concerne aos diferentes itens para a formação da Perspectiva Abrangente dos Clientes. De acordo com cada prioridade, seria também estabelecido um fator de ponderação cujo valor (ou peso) refletiria sua importância em relação aos outros, mas de forma que a soma dos mesmos fosse 100. Com base nesta pondera- 
ção, poder-se-ia produzir uma configuração de ativo ou passivo relativo à Perspectiva Abrangente dos Clientes que estaria associada a cada um de seus itens formadores (satisfação, fidelidade, retenção, captação e lucratividade dos clientes), ao multiplicarse a porcentagem de avanço ou retrocesso de cada item pelo valor (ou peso) relativo de sua contribuição ao Balanço da Perspectiva Abrangente dos Clientes (BPAC). O peso relativo de cada contribuição determina a taxa de conversão das medidas USC, UFC, URC, UCC e ULC para a unidade de perspectiva abrangente dos clientes (UPAC).

\section{Tabela 2 - Síntese do balanço da perspectiva abrangente dos clientes de uma empresa hipotética.}

\begin{tabular}{|c|c|c|c|c|c|c|}
\hline \multirow{2}{*}{$\begin{array}{l}\text { TIPO DE PERSPECTIVA } \\
\text { DOS CLIENTES }\end{array}$} & \multirow[b]{2}{*}{ PR } & \multirow[b]{2}{*}{ FP ou PE } & \multicolumn{2}{|c|}{ RES. 1998-1999 } & \multirow[b]{2}{*}{ APAC } & \multirow[b]{2}{*}{ PPAC } \\
\hline & & & GSPAC & GIPAC & & \\
\hline Satisfação & 1 & $40 / 100$ & 192,45 & 181,00 & 76,98 & 72,40 \\
\hline Fidelidade & 2 & $20 / 100$ & 180,73 & 180,03 & 36,15 & 36,01 \\
\hline Retenção & 3 & $20 / 100$ & 174,17 & 171,21 & 34,83 & 34,24 \\
\hline Captação & 4 & $10 / 100$ & 166,04 & 162,53 & 16,60 & 16,25 \\
\hline Lucratividade & 5 & $10 / 100$ & 173,65 & 172,39 & 17,37 & 17,24 \\
\hline \multicolumn{5}{|c|}{ Grau de Satisfação quanto a Perspectiva Abrangente dos Clientes (UPAC) } & 181,93 & \\
\hline \multicolumn{6}{|c|}{ Grau de Insatisfação quanto a Perspectiva Abrangente dos Clientes (UPAC) } & 176,14 \\
\hline \multicolumn{6}{|c|}{ Superávit na Satisfação quanto a Perspectiva dos Clientes da Empresa (UPAC) } & 5,79 \\
\hline \multicolumn{5}{|c|}{ Totalização } & 181,93 & 181,93 \\
\hline
\end{tabular}

PR - Prioridade; FP - Fator de Ponderação; PE - peso; E(\%) - Avanço ou retrocesso da avaliação (\%); GSPAC - Grau de Satisfação quanto a Perspectiva Abrangente dos Clientes (em UPAC); GIPAC - Grau de Insatisfação quanto a Perspectiva Abrangente dos Clientes (em UPAC); APAC - Ativo relacionado à Perspectiva Abrangente dos Clientes; PPAC - Passivo relacionado à Perspectiva Abrangente dos Clientes; UPAC - Unidade de Perspectiva Abrangente dos Clientes.

A Tabela 2 apresenta um exemplo dos indicadores relativos aos itens da perspectiva abrangente dos clientes, resultantes de enquêtes realizadas por uma empresa hipotética. Deve-se destacar que os indicadores nas colunas dos resultados refletem o esquema utilizado na ponderação da importância das contribuições da satisfação, fidelidade, retenção, captação e lucratividade dos clientes da empresa por parte de quem está preparando o Balanço da Perspectiva Abrangente do Cliente (BPAC). Uma análise do balanço mostra que à perspectiva abrangente dos clientes no biênio 1998-1999 teve ativos superiores aos passivos em todos os indicadores, o que gerou um superávit na satisfação quanto a perspectiva abrangente dos clientes. No entanto, este superávit (medido em UPAC) foi pequeno, menos de $5 \%$ do ativo gerado.

Deve-se enfatizar que o Balanço da Perspectiva Abrangente do Cliente (BPAC) embora deva ser preparado anualmente, só tem sentido pela comparação dos resultados de seus indicadores no período de dois anos. Claro que as enquêtes aplicadas du- rante cada período de dois anos devem ser as mesmas, daí o extremo cuidado que se deve dispensar à sua elaboração. Caso as enquêtes mudem, deve ser determinado um índice de equalização, o qual permitirá a comparação dos ativos e passivos referentes aos indicadores da perspectiva abrangente do cliente de um biênio com outros de um novo biênio, no qual as enquêtes tenham sofrido mudanças.

\section{CONCLUSÕES E PERSPECTIVAS}

O método quantitativo para avaliação da satisfação dos clientes proposto neste trabalho constitui um avanço significativo na busca da generalização do modelo venerável da contabilidade financeira no sentido de incorporar a avaliação de ativos intangíveis para a empresa, que no caso foi a satisfação do cliente. Foi demonstrado ser factível o seu emprego para a obtenção de informações quantitativas sobre fidelidade, retenção, captação e lucratividade dos clientes da empresa no mesmo esquema proposto para o caso da satisfação dos clientes. 
O resultado disso abre portas para a possibilidade da preparação do que seria denominado de $\mathrm{Ba}$ lanço da Perspectiva Abrangente dos Clientes (BPAC) de uma empresa, que conteria informações quantitativas sobre a perspectiva abrangente dos clientes. O Balanço da Perspectiva Abrangente dos Clientes (BPAC) aqui proposto não apresenta o desconforto de ser apenas mais um demonstrativo técnicogerencial entre os existentes, mas se configura como um documento de caráter contábil em que a essência de sua preparação se remete às partidas dobradas, marco fundamental na Ciência Contábil. Ele possibilita uma determinação analítica da performance de uma empresa quanto à perspectiva dos clientes, a partir de indicadores cuidadosamente escolhidos, os quais refletem diferentes aspectos quantitativos $\mathrm{e}$ qualitativos das ações desenvolvidas/avaliadas pelas empresas. A implementação do BPAC definitivamente possibilita o advento do que seria uma Auditoria da Perspectiva dos Clientes, específica sobre ativos e passivos da empresa decorrentes de sua mensuração através do BPAC.
Finalmente, o BPAC é um documento que, indubitavelmente, torna indispensável a participação de contadores na sua preparação. Mas não a daquele antigo profissional desatualizado, que não insere a Ciência Contábil no contexto da nova era da informação que vivenciamos atualmente, mas sim o que tem uma visão da empresa não mais como um conjunto de números que refletem os ativos e passivos tradicionais da contabilidade corriqueira. Trata-se aqui da visão integrada dos negócios, onde correlações de longo alcance são geridas por fenômenos econômicos, as de médio alcance por fenômenos gerenciais, ambas totalmente influenciadas pelas correlações de curto alcance geridas pelos processos contábeis. Fazse mister que o profissional contábil que venha a tomar para si a preparação do BPAC de empresas, tenha conhecimentos básicos econômicos e gerenciais modernos, que o capacitem a implementar as enquêtes com todas as questões relevantes para o estabelecimento dos indicadores relativos à perspectiva dos clientes.

\section{REFERÊNCIAS BIBLIOGRÁFICAS}

ADMS, C. e ROBERT, P. You are what you measure. Manufacturing Europe, Sterling Publications Ltd, p. 504-507,1993.

ATKINSON, A.; BANKER, R. D.; KAPLAN, R. S. e Young, S. M. Contabilidade gerencial. São Paulo: Atlas, 2000.

FREIRE, F. de S; CRISÓSTOMO, V. L; BOTELHO, D. R. e NUNES, F. M. Um método quantitativo para avaliação da satisfação dos clientes. Anais do VII Congresso Internacional de Custos, Léon, Espanha, juIho de 2001.

HRONEC, S. M. e Arthur A \& Co. Vital sings - using quality, time and cost performance measurement to chart you company's future. New York: American Management Association, 1993.
KAPLAN, R. S. e NORTON, D. P. Organização orientada para a estratégia: como as empresas que adotam o balanced scorecard prosperam no novo ambiente de negócios. Rio de Janeiro: Campus, 2000.

KAPLAN, R. S. e NORTON, D. P. A estratégia em ação - balanced scorecard. Rio de Janeiro: Campus, 1997.

KAPLAN, R. S. e NORTON, D.P. The balanced scorecardmeasures that drive performance. Boston: Havard Business Review, janeiro-fevereiro, p. 71-79, 1992.

NORTON, D. P. Medir a criação de valor. uma tarefa possível. Management, ํㅡㄴ 24, Ano 4, janeiro-fevereiro, p. 88-94, 2001.

IJIRI, Y. The foundations of accounting measurement. New Jersey: Prentice-Hall, Inc. Englawood Cliffs, 1997. 